\title{
Canine leishmaniosis caused by Leishmania major and Leishmania tropica: comparative findings and serology
}

\author{
Gad Baneth ${ }^{*}$, Daniel Yasur-Landau, Matan Gilad and Yaarit Nachum-Biala
}

\begin{abstract}
Background: Infection and clinical disease associated with Leishmania major and Leishmania tropica, two common agents of human cutaneous leishmaniosis, have rarely been reported in dogs. This study describes dogs infected with these Leishmania spp. prevalent in the Middle East and North Africa, and compares the serological response of dogs infected with Leishmania infantum, L. major or L. tropica to whole promastigote antigen enzyme-linked immunosorbent assay (ELISA) of each species and to rK39 dipstick.
\end{abstract}

Results: Leishmania major infection in a 5-month-old male dog was associated with alopecic and ulcerative periocular and limb skin lesions which responded to allopurinol treatment. Infection was detected by skin and blood polymerase chain reaction (PCR) and confirmed by DNA sequencing but the dog was seronegative. Leishmania tropica infection was detected in a 3-month-old female dog co-infected with Babesia vogeli and Anaplasma platys and with no skin lesions. PCR and DNA sequencing of the blood and parasite culture were positive for $L$. tropica. Sera from 11 dogs infected with L. infantum, L. major or L. tropica were reactive with all three Leishmania spp. antigens except for sera from a dog with $L$. major infection. No significant differences were found between reactivity of dog sera to the antigen of the infecting species, or to the other Leishmania spp. antigens. Sera from dogs infected with $L$. infantum and $L$. tropica were positive with the rK39 antigen kit, while dogs with L. major infection were seronegative.

Conclusions: Skin lesions in L. major infected dogs from this study and previous reports $(n=2)$ were ulcerative and located on the muzzle, feet and foot pads and not associated with generalized lymphadenomegaly and splenomegaly. In previous $L$. tropica infections, skin lesions were proliferative mucocutaneous in young dogs $(n=2)$, or associated with widespread dermatitis, lymphadenomegaly and splenomegaly in older dogs with similarity to L. infantum infection ( $n$ $=2$ ). This study suggests that ELISA serology with whole promastigote antigen is not distinctive between $L$. infantum, $L$. major and L. tropica canine infections and that some L. major infections are not seropositive. PCR with DNA sequencing should be used to discriminate between canine infections with these three species.

Keywords: Leishmania major, Leishmania tropica, Cutaneous leishmaniosis, Canine leishmaniosis, Israel, Co-infections

\section{Background}

Leishmania major and Leishmania tropica cause human cutaneous leishmaniosis in Asia and Africa with infringement of $L$. tropica also into Greece in southern Europe [1, 2]. In the Middle East and Israel, these two Leishmania spp. are common causes of human infection with wildlife mammal reservoirs, with Phlebotomus papatasi as the sand fly vector for L. major, and Phlebotomus

\footnotetext{
* Correspondence: gad.baneth@mail.huji.ac.il

School of Veterinary Medicine, Hebrew University, P.O. Box 12, Rehovot 76100, Israel
}

(c) The Author(s). 2017 Open Access This article is distributed under the terms of the Creative Commons Attribution 4.0 International License (http://creativecommons.org/licenses/by/4.0/), which permits unrestricted use, distribution, and reproduction in any medium, provided you give appropriate credit to the original author(s) and the source, provide a link to the Creative Commons license, and indicate if changes were made. The Creative Commons Public Domain Dedication waiver (http://creativecommons.org/publicdomain/zero/1.0/) applies to the data made available in this article, unless otherwise stated. $[1,3,4]$. Domestic dogs have been shown to suffer from clinical disease associated with infection with these two Leishmania spp., although L. major and L. tropica are considered rare causes of leishmaniosis in dogs in the Old World, compared to L. infantum, and a study from southeastern Iran has detected clinical L. tropica infection verified by PCR in only two of $471(0.4 \%)$ dogs in the endemic Kerman region [5]. Likewise, descriptions of the clinical characteristics and laboratory test findings of these two canine infections are scant and there are no established protocols for their detection or knowledge on what should 
be expected in serological testing of these infections. The aim of this study was to describe additional clinical canine cases of L. major and L. tropica infections, compare them to previous ones $[6,7]$ and study the serological response to infection using antigens of three different Leishmania spp.

\section{Methods}

Dogs

Dogs diagnosed with $L$. tropica or $L$. major infection during 2015-2016 at the Hebrew University School of Veterinary Medicine (HUSVM) laboratory for vectorborne infectious diseases were included in the study. Data on history, clinical signs, hematology and serum biochemistry and specific tests for the diagnosis of leishmaniosis were collected from each case and samples were taken for parasitological detection of the disease.

\section{Leishmania PCR}

Leishmania detection was performed by realtime PCR using primers JW11/JW12 targeting a 120 bp sequence of the Leishmania short fragment from the kinetoplast minicircle [8]. Additional detection and identification was carried out by high resolution melt (HRM)-PCR using primers ITS-219 F and ITS-219R to amplify a 265 bp fragment of the Leishmania ribosomal operon internal transcribed spacer 1 (ITS1) region and then evaluated by HRM analysis as previously described [9]. PCR was performed using the StepOnePlus real-time PCR thermal cycler (Applied Biosystems, Foster City, CA, USA) as previously described [10]. DNA samples extracted from parasite promastigote cultures of $L$. infantum, $L$. tropica and $L$. major were used as positive controls for each corresponding PCR and DNA from colony-bred dogs negative by PCR for vector-borne pathogens was used as a negative control. A non-template control (NTC) with the same reagents described above but without DNA was added to each PCR to rule out contamination.

All positive PCR products were sequenced using the BigDye Terminator v3.1 Cycle Sequencing Kit and an ABI PRISM 3100 Genetic Analyzer (Applied Biosystems, Foster City, CA, USA) at the Center for Genomic Technologies, Hebrew University of Jerusalem, Israel. DNA sequences were evaluated with the ChromasPro software version 2.1.1 (Technelysium Pty Ltd., Australia) and compared for similarity with sequences available in GenBank, using the BLAST program (http://www.ncbi.nlm.nih.gov/BLAST/).

\section{Parasite culture}

Leishmania promastigotes grown in culture were prepared as crude antigen for serology as previously described [11]. The strains used for antigen production were L. infantum MCAN/IL/1994/LRC-L639, L. tropica MHOM/IL/2005/ LRC-L1239 and L. major MHOM/TM/1973/5ASKH. The antigens were prepared for serological testing carried out in 96-well enzyme-linked immunosorbent assay (ELISA) plastic plates coated with $1.5 \mu \mathrm{g}$ protein per well.

\section{Serology}

Serology for the dog cases was performed for the initial diagnostic procedures using L. infantum antigen by ELISA as previously described [11]. Subsequently, ELISA serology was performed with three different Leishmania spp. antigens: L. infantum, L. major and L. tropica for each serum sample available from the $L$. major- and L. tropicainfected dogs, including also for sera from previously described canine L. tropica and L. major cases $[6,7]$. In addition, sera from eight dogs with clinical L. infantum disease diagnosed in the HUSVM and confirmed by HRM-PCR [9] and DNA sequencing were also evaluated. All sera were tested for reactivity with each of the three Leishmania spp. antigens at the same time. All dog sera, tested at 1:100 dilutions, were incubated with one of the three different leishmanial antigen coated plates for $1 \mathrm{~h}$ at $37{ }^{\circ} \mathrm{C}$. The plates were then washed with $0.1 \%$ Tween 20 in $50 \mathrm{mM}$ phosphate-buffered saline (PBS), $\mathrm{pH} 7.2$, and incubated with Protein A conjugated to horseradish peroxidase (1:10,000 dilution; Zymed Laboratories, Inc., San Francisco, CA, USA) for $1 \mathrm{~h}$ at $37{ }^{\circ} \mathrm{C}$. Excess conjugate was removed by extensive washing in PBS-Tween and the plates were developed by addition of the substrate 2,29-azino-di-3-ethylbenzthiazoline sulfonate (ABTS) (Boehringer Mannheim, Mannheim, Germany). Each plate was read when the absorbance (lambda $=405 \mathrm{~nm}$ ) of the positive canine reference serum reached a value between 1.2-1.4. Dilutions of positive and negative reference dog sera were included on each plate to monitor interassay variation.

All results were adjusted to the highest values obtained from serum from a dog infected with the same species of Leishmania as the plate coating antigen, e.g. for a plate coated with $L$. infantum antigen, all results were adjusted to serum from a dog infected with L. infantum. Each serum was run in triplicate and the final optical density (OD) value was calculated as the average of readings. OD values were adjusted for each antigen separately and eventually sera from each dog infected with a certain Leishmania species were compared with readings of its reactivity with other Leishmania spp. antigens and with sera of other dogs infected by the same species, in order to investigate if using a homologous Leishmania antigen is beneficial for the diagnosis of the infecting species. Serological cut-off values for each Leishmania sp. were calculated based on two standard deviations above the mean OD value of readings from eight control sera from seronegative and PCR-negative dogs.

Antibodies reactive with the recombinant antigen rK39 were tested using the Kalazar Detect dipstick kit 
(InBios International Inc., Seattle, Washington, USA) according to the manufacturer's instruction.

\section{Statistical analysis}

All data distributed normally as tested by the ShapiroWilk test. A One-Way ANOVA was used to determine the mean differences between OD values obtained for serum of dogs infected with the same Leishmania spp. or with different Leishmania spp. antigens. Statistical analysis was performed using the SPSS $^{\circ} 21.0$ software (IBM, Armonk, New York, USA).

\section{Results}

\section{Clinical cases}

Case no. 1

A 5-month-old mixed breed dog male castrated dog that had been adopted from an animal shelter in Jerusalem, Israel, was admitted to a veterinary clinic with lethargy, anorexia and dermatologic abnormalities. The dog had suffered from skin lesions on its face and legs when it was brought to the shelter 3 weeks earlier. The skin lesions consisted of unilateral periocular alopecia with ulceration, scales and serous discharge around the right eye (Fig. 1a), ulceration in the left hind foot pad and ulcerative scaly lesions on the left front leg over the carpus (Fig. 2) and left hind leg over the tarsus. Physical examination at the veterinary clinic was otherwise normal. A complete blood count $(\mathrm{CBC})$ indicated mild normocytic normochromic anemia with a packed cell volume (PCV) of 31\% (reference range 32-55\%) and serum biochemistry panel was within normal limits. Skin scrapings from the lesions were negative for Demodex spp., and culture for dermatophytes was also negative. ELISA serology for L. infantum submitted to the HUSVM was negative, however, a skin biopsy taken from the eye lesion and submitted for PCR for Leishmania spp. at the HUSVM and was positive for Leishmania by HRM-PCR with a melt curve pattern compatible with $L$. major which was confirmed by sequencing of a 240 bp DNA PCR product that had 100\% identity to L. major (GenBank: KP773413.1) and was deposited in GenBank (KY524299). The dog was referred to the HUSVM teaching hospital for further diagnosis and follow up. It was admitted a month after its initial visit to the referring veterinarian and on physical examination skin lesions on the feet were in a similar state to that found a month earlier but the periocular lesion
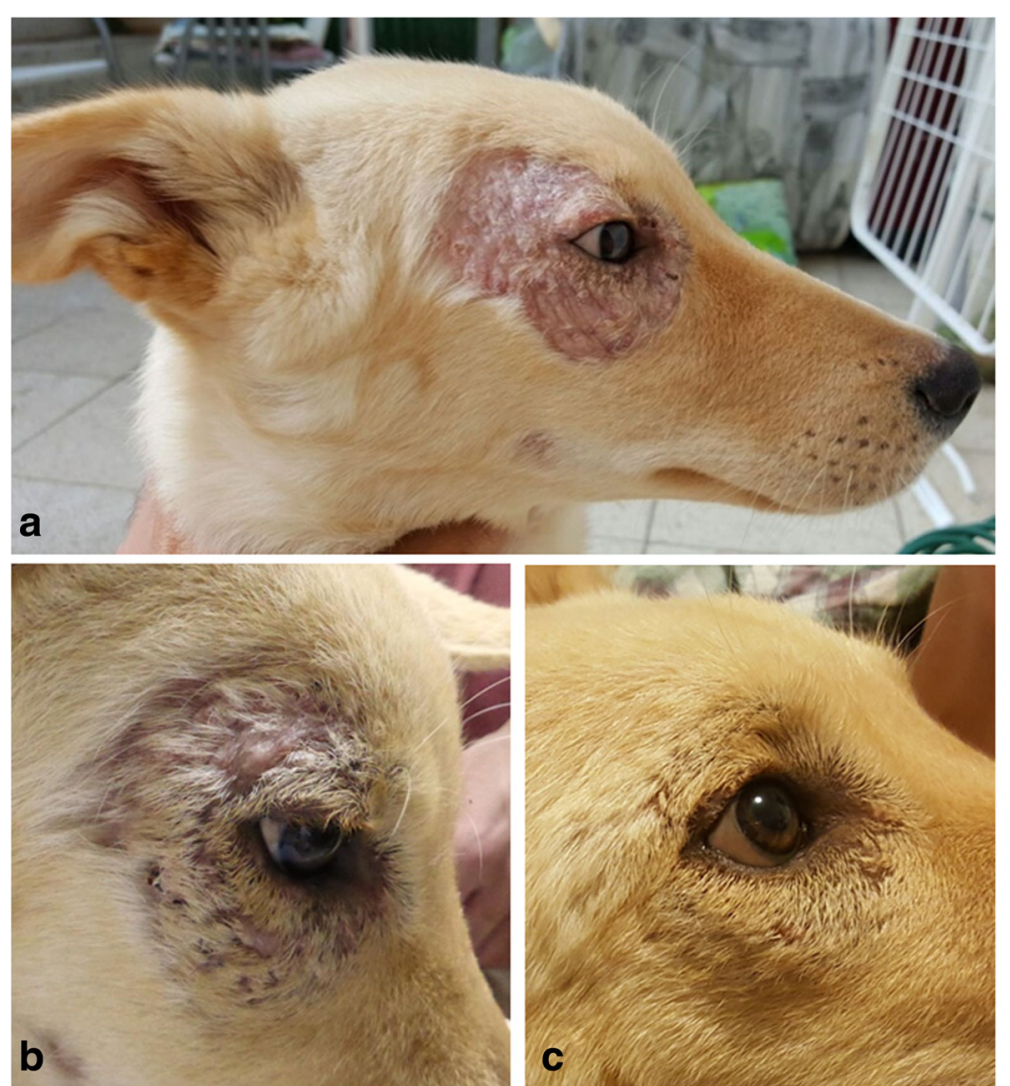

Fig. 1 Cutaneous periocular Leishmania major skin lesion in dog case no. 1 with changes over time: a on initial diagnosis at 6 months of age; $\mathbf{b}$ at 7 months of age on the day of begining allopurinol treatment. Some healing and growth of hair can be observed even before treatment; c 7 weeks after begining treatment 


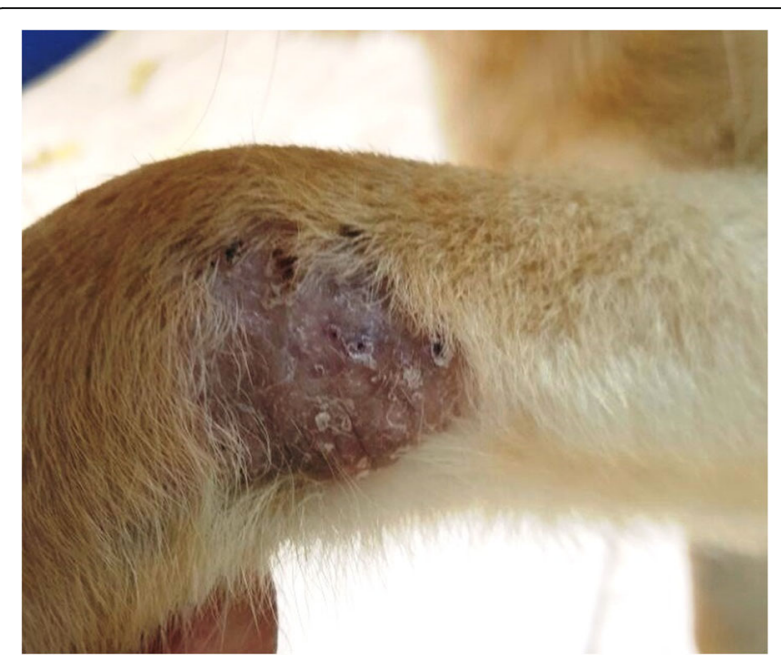

Fig. 2 Cutaneous skin lesion over the carpus in dog case no. 1 infected with Leishmania major. Image taken at 6 months of age prior to treatment

appeared to improve with the disappearance of the serous discharge and some regrowth of hair (Fig. 1b). Complete blood count (CBC) showed worsening of the normoncytic normochromic anemia with a PCV of $25 \%$. Serum biochemistry and urinalysis were within normal limits. Left and right conjunctival swabs, prescapular lymph node aspirates and urine were negative for Leishmania by kDNA PCR [8]; however blood was positive by the same PCR protocol and confirmed by DNA sequencing with the closest identity to L. major (GenBank: EU370907.1). ELISA serology for $L$. infantum was negative again. Treatment with $10 \mathrm{mg} / \mathrm{kg}$ of allopurinol orally twice daily was started and the owners were instructed to apply a topical insecticide against sand fly bites on the dog. The dog was rechecked at the HUSVM teaching hospital 7 weeks after the beginning of treatment. The dog's skin condition had improved and the periocular lesion had almost disappeared under dense regrowth of hair covering moderately thickened small skin scars (Fig. 1c). The skin lesions over the left tarsus and carpus have also healed and were almost unapparent. The dog was no longer anemic with a PCV of $39 \%$ and serum biochemistry was within normal limits. PCR of the blood was negative and serology was repeatedly negative. The owners were instructed to continue allopurinol treatment and recheck the dogs 3 months later.

\section{Case no. 2}

A 3-month-old stray female intact pup was admitted to the HUSVM teaching hospital from Tel Aviv in Israel with a complaint of lethargy. On physical examination the dog was thin with a decreased body condition score (2/9), a normal rectal body temperature and with mild serous ocular discharge. On CBC the dog was anemic (PCV 22\%) with a regenerative anemia, and moderately thrombocytopenic $\left(110 \times 10^{9}\right.$ thrombocytes/l; reference $\left.150-400 \times 10^{9} / 1\right)$. Stained blood smear evaluation revealed inclusions in thrombocytes suspected as Anaplasma platys morulae and large form Babesia sp. organisms in erythrocytes. PCR for Anaplasma sp. and for Babesia sp. was carried out using the piroplasmid and EC-16S primers for Babesia and Anaplasma/Ehrlichia spp., respectively, as previously described $[12,13]$. Both PCRs were positive and following DNA sequencing it was confirmed that the dog was infected with Babesia vogeli (99\% identical to GenBank HQ662635.1) and A. platys (99\% identical to GenBank JQ976643.1). No serum was available from this dog for serology. The dog was treated with doxycycline at $10 \mathrm{mg} / \mathrm{kg}$ P.O. daily for 14 days against anaplasmosis, and imidocarb dipropionate at a reduced dose of $2.5 \mathrm{mg} / \mathrm{kg}$ due to the dog's young age by single I.M. injection against the babesial infection with premedication of atropine at $0.05 \mathrm{mg} / \mathrm{kg}$ I.M. to prevent the possible cholinergic effects of imidocarb dipropionate. In addition, the dog was treated with an antibiotic eye ointment containing $5 \%$ tetracycline.

The dog was hospitalized, received I.V. fluid transfusion and followed up clinically. It was discharged after 4 days when it improved clinically and regained normal activity and was adopted by new owners that were instructed to come for a recheck and repeated imidocarb dipropionate injection after 10 days but did not return for medical treatment and refused further contact.

Pre-treatment blood from the dog was submitted to the laboratory to attempt isolation of $A$. platys, which has not been grown successfully in culture to date. It was initially transferred to a temporary medium, which has a similar composition to the medium used as a basis for culture of Leishmania, before seeding in a cell culture which was not carried out eventually as Leishmania was detected in the transfer medium. Essentially, $100 \mu \mathrm{l}$ of blood was incubated in an equal volume of minimum essential medium (MEM) (Sigma, Saint Louis, USA)/Leibovitz's L15 medium (Gibco, Carlsbad, CA, USA), supplemented with 20\% fetal calf serum (Biological industries, Beit Haemek, Israel), 10\% tryptose phosphate broth solution (TPB, Sigma-Aldrich, Saint Louis, USA), $1 \%$ penicillin-streptomycin (Sigma-Aldrich, Saint Louis, USA) and 1\% L-glutamine (Biological industries, Beit Haemek, Israel). After $2 \mathrm{~h}$, medium was removed and replaced by fresh medium and incubated at $29^{\circ} \mathrm{C}$. When the medium was tested by microscopy after 7 days, it contained propagating organisms which appeared similar in shape to Leishmania spp. promastigotes and indeed were identified as L. tropica by HRM-PCR and DNA sequencing (100\% identical to GenBank GU561643.1). This L. tropica sequence was deposited in GenBank (KY524300). To verify Leishmania spp. infection in the dog, blood kept from the dog's CBC was tested by PCR for Leishmania and was also positive by kDNA PCR [8]. 
This diagnosis was surprising since the dog did not show clinical signs that were suspicious of leishmaniosis such as skin lesions.

\section{Comparative serology}

Altogether 19 sera samples from 11 dogs infected with one of the three Leishmania spp. studied were available: eight from individual dogs with L. infantum; six from two dogs with $L$. major infection (four from one dog taken over a five months period and two from a second dog taken over three months); and five from a dog with $L$ tropica infection taken at different time points over 26 months (see Additional file 1: Table S1). The serological cut-off values calculated from seronegative dogs were $0.18,0.068$ and 0.101 for L. infantum, L. major and L. tropica, respectively. Sera from all dogs reacted positively with all three Leishmania species antigen except for the two samples from case no. 2 with $L$. major infection described in this study which did not react with $L$. major antigen nor with the two other leishmanial species antigens (Additional file 1: Table S1). After omitting dog no. 2's negative results, no significant differences $\left(F_{(2,23)}\right.$ $=0.72, P=0.498$ for $L$. infantum antigen; $F_{(2,11)}=0.118$, $P=0.890$ for $L$. major antigen; $F_{(2,14)}=0.336, P=0.721$ for $L$. tropica antigen) were found between reactivity of dog sera to the antigen of the infecting species as expressed in OD, and reactivity to the other two Leishmania species antigens (Figs. 3, 4 and 5).

Sera of dogs infected with L. infantum and L. tropica reacted positively with the rK39 antigen kit, while the sera of dogs with $L$. major infection were negative by this antigen kit.

\section{Discussion}

This study describes unique cases of dog infection with L. major and L. tropica, two common agents of cutaneous leishmaniosis in people in the Middle East and North Africa, albeit rarely reported as associated with disease in dogs $[1,3]$. These cases provide additional clinical, diagnostic and therapeutic information on these infections in dogs and add to other reports based on molecular or enzymatic biochemical characterization of clinical canine $L$. major $[7,14-16]$ and $L$. tropica infections [5, 6, 17-19]. In addition, they provided an opportunity to compare the reactivity of serum from dogs to different leishmanial antigens and evaluate the value of serological testing in canine infection with $L$. major and L. tropica.

The dogs described in this study and other molecularly characterized reports of $L$. major and L. tropica canine infections $[6,7,19]$ have described animals younger than 1-year-old infected with L. major [7] and both

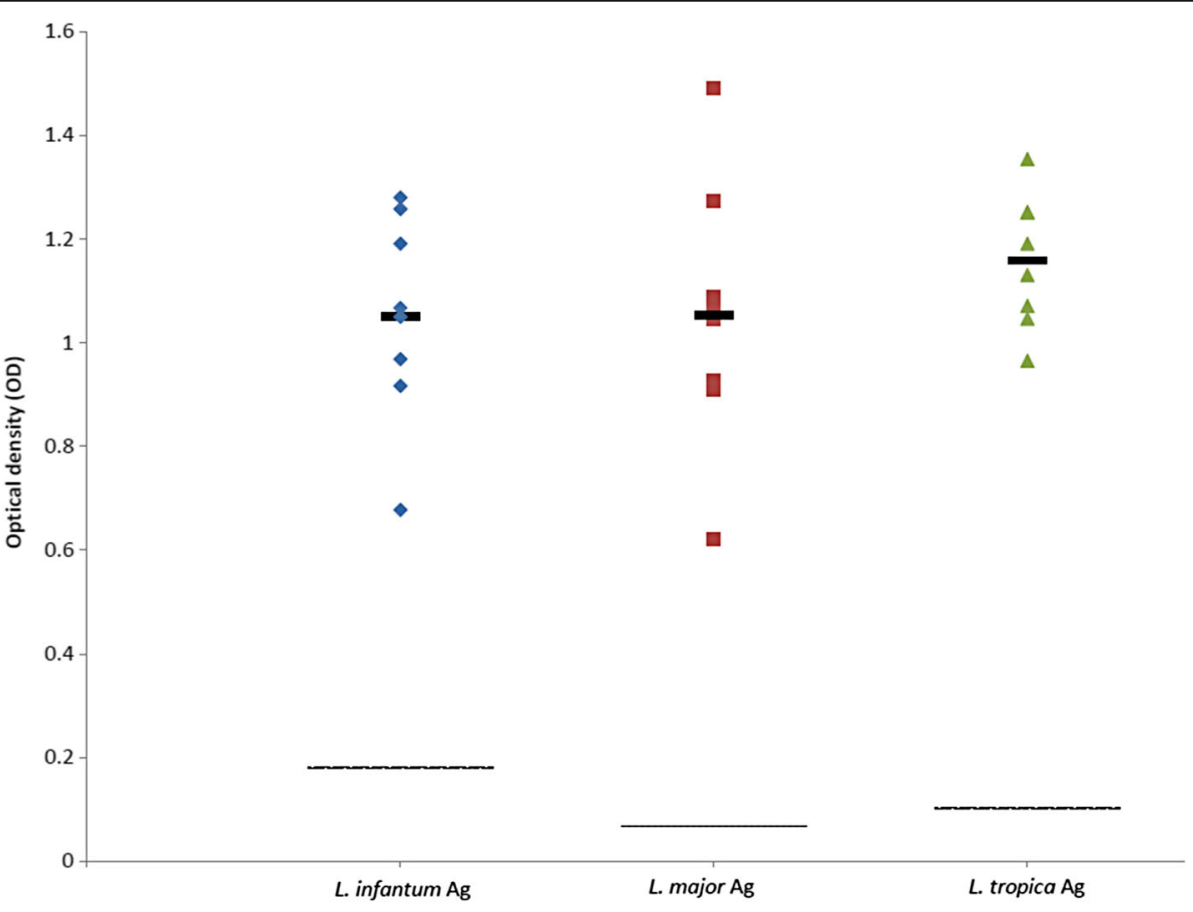

Fig. 3 Scatter plot showing reactivity of sera tested by ELISA from eight dogs infected with Leishmania infantum. Seroreactivity was tested with antigens of L. infantum, L. major and L. tropica. Y-axis shows calibrated optical density (OD). The thick black line represents the mean value. The intermittent line represents the serological cut-off value. No significant differences were found between OD readings of the same sera with the different antigens $\left(F_{(2,23)}=0.72, P=0.498\right)$ 


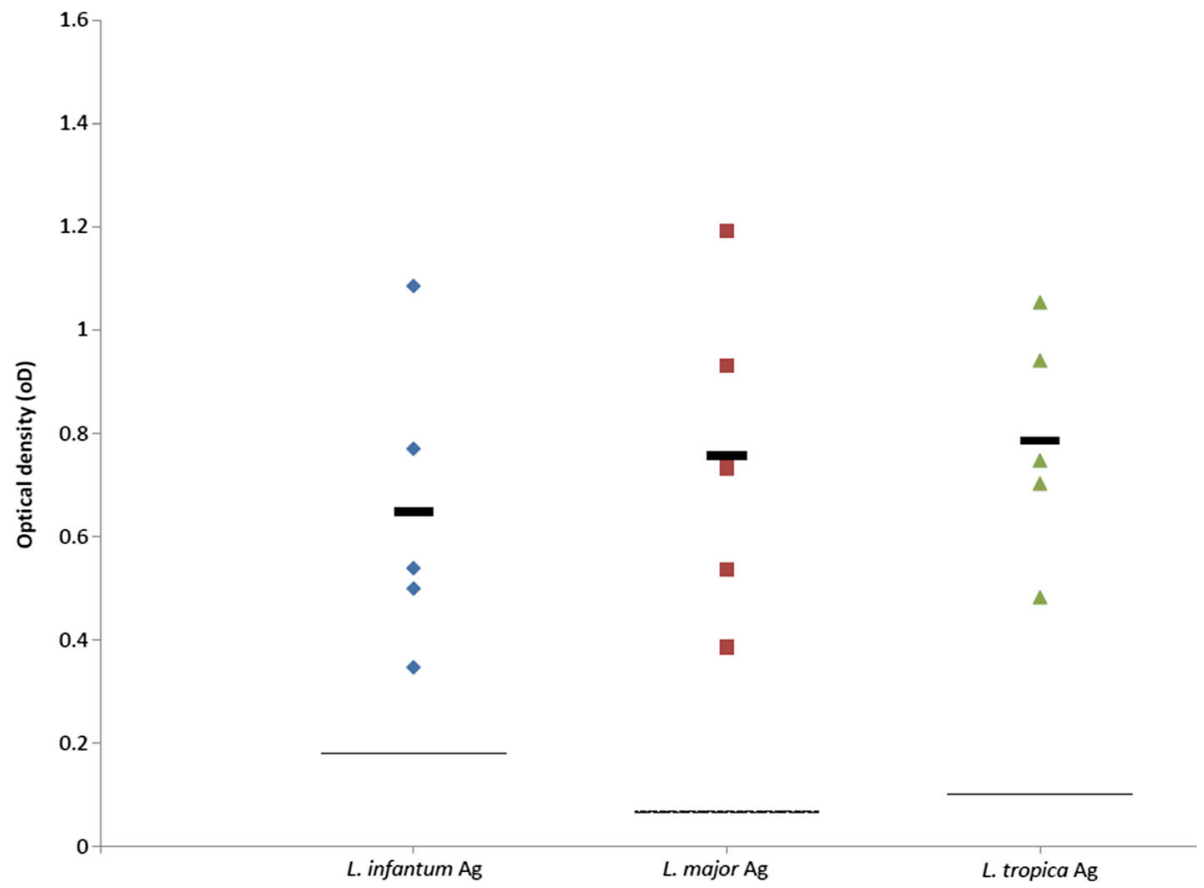

Fig. 4 Scatter plot showing reactivity of five sera tested by ELISA from two dogs infected with Leishmania tropica. Seroreactivity was tested with antigens of L. infantum, L. major and L. tropica. Y-axis shows calibrated optical density (OD). The thick black line represents the mean value. The intermittent line represents the serological cut-off value. No significant differences were found between OD readings of the same sera with the different antigens $\left(F_{(2,14)}=0.336, P=0.721\right)$

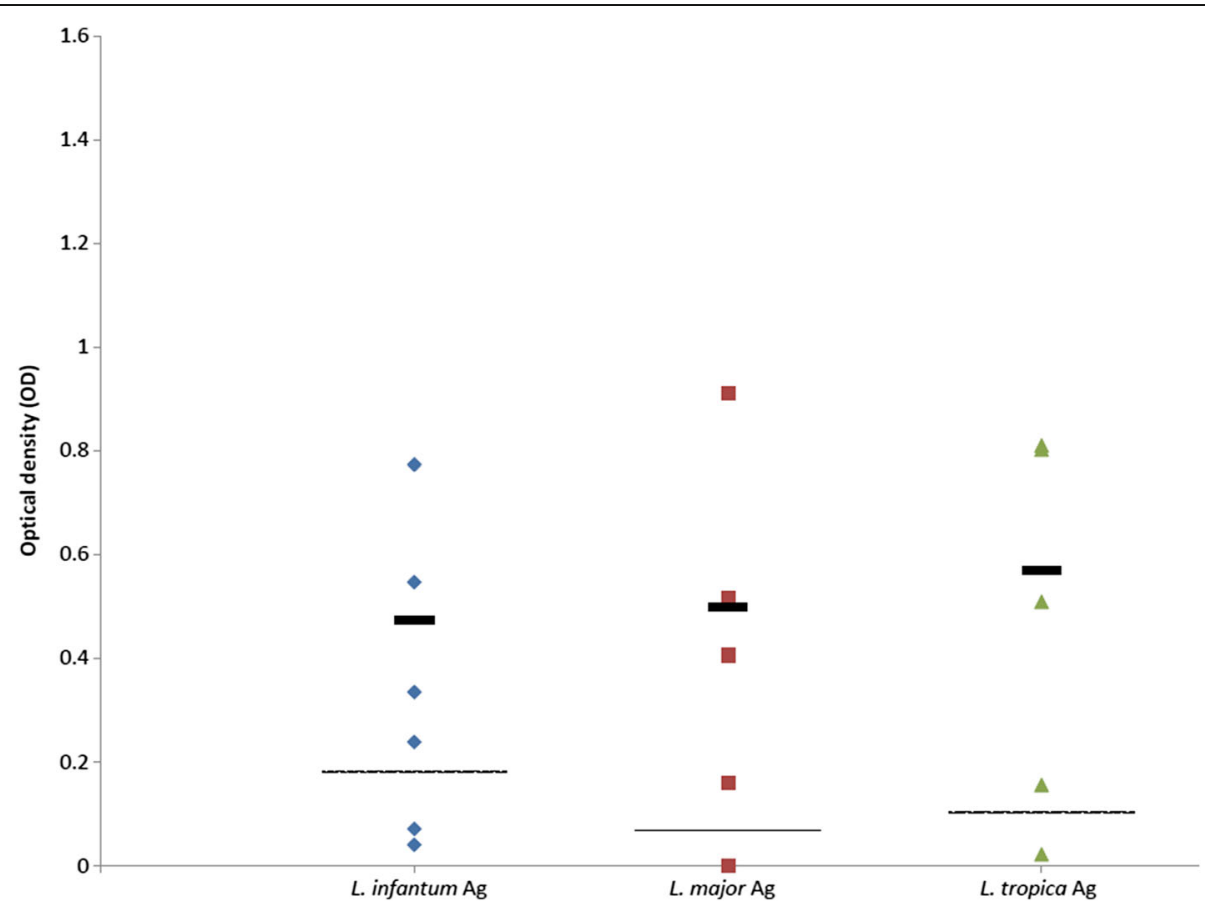

Fig. 5 Scatter plot showing reactivity of six sera tested by ELISA from two dogs infected with Leishmania major. Seroreactivity was tested with antigens of L. infantum, L. major and L. tropica. Y-axis shows calibrated optical density (OD). The thick black line represents the mean value. The intermittent line represents the serological cut-off value. Samples 6835 and 0026 were omitted from the mean calculation due to dog case no. 2 being seronegative. No significant differences were found between OD readings of the same sera with the different antigens $\left(F_{(2,11)}=0.118, P=0.824\right)$ 
young $[6,19]$ and older dogs above 5 years of age infected with L. tropica [5]. Skin lesions in L. major infected dogs from this study and a previous report [7] were ulcerative and located on the muzzle, feet and foot pads and not associated with generalized lymph node enlargement and palpable splenomegaly. In contrast, in L. tropica infection, skin lesions were mucocutaneous and proliferative in two cases of young dogs $[6,19]$, or associated with pustular dermatitis, lymphadenomegaly and splenomegaly [5]. Older descriptions of $L$. tropica-infected dogs diagnosed based on culture and enzymatic characterization describe dermatitis with facial papules and no other clinical manifestations in seven dogs from Morocco [20] or a severe disease, with poor body condition, skin and internal organ pathology similar to canine viscerocutaneous leishmaniosis caused by $L$. infantum $[17,18]$.

The descriptions of only skin lesions in canine $L$. major infection while canine $L$. tropica infection may manifest as a skin disease which may in some cases also disseminate to visceral organs and cause generalized disease, are in agreement with these diseases in people where $L$. major causes cutaneous disease but $L$. tropica is also involved with human visceral leishmaniosis [21, 22].

The hematological and serum biochemistry findings in canine L. major infection described here (case no. 1) indicated a mild anemia with no serum biochemistry abnormalities. A previous report of $L$. major infection [7] described no CBC and serum chemistry abnormalities. The laboratory findings for the L. tropica infected dog (case no. 2) with anemia, leukopenia and mild thrombocytopenia were probably affected by the infections with $B$. vogeli and $A$. platys and should not be interpreted as necessarily associated with $L$. tropica infection. A previous case of $L$. tropica had a mild leukocytosis and eosinophilia with no anemia [6], while an Iranian young dog with L. tropica infection had a reported normal hemogram [19].

Overall, the hematology and serum biochemistry findings from L. major and L. tropica infected dogs suggest that they present a different picture from the typical findings of hyperglobulinemia, hypoalbuminemia and anemia found in dogs with generalized canine leishmaniosis caused by L. infantum [23]. This may be due to the visceralization of $L$. infantum with changes in internal organs associated with this chronic infection.

Detection of $L$. major DNA by PCR in blood was successful in the dog reported here and negative on multiple dates in a previous report [7] where the prescapular lymph node was positive. In L. tropica infected dogs, blood PCR was positive in the dog reported here and parasite culture was grown out of the dog's blood; however, blood PCR was negative in a previous report on a dog with mucocutaneous leishmaniosis [6]. Therefore, it can be concluded blood PCR may be positive in canine L. major and L. tropica infections; however, it is probably not a sufficiently sensitive and reliable test for confirming these infections. Blood PCR is also not an optimal test for confirming canine $L$. infantum infection and bone marrow or lymph node PCR are preferred [23].

ELISA serology with crude promastigote antigen was not found to be distinctive between Leishmania species in dogs in this study as there was no significant association between the leishmanial species infecting the dogs and their seroreactivity to its antigen when compared to antigen of other species. Furthermore, the dog with $L$. major infection (case no. 1) was seronegative for all antigens in contrast to a dog with L. major infection described in a previous study [7] whose serum was reactive with the antigens of all Leishmania spp. in this study.

Canine serological cross-reactivity between Leishmania spp. antigens has been described before and dogs infected with L. infantum (syn. Leishmania chagasi) from Brazil have been shown to respond to antigen from $L$. major-like antigen by ELISA [24] and also to L. braziliensis antigen [25]. Furthermore, sera from Brazilian L. infantum (chagasi)-infected dogs was reactive by western immunoblotting with several antigens from whole promastigote antigens of Leishmania guyanensis, Leishmania amazonensis and L. braziliensis [26]. Therefore, cross-reactivity amongst the Old World species of L. major, L. tropica and $L$. infantum as found in dogs in the current study is not surprising, and it is important to note that ELISA has not been found distinctive for the infecting Leishmania species with regard to the OD level of reactivity in this study.

The use of recombinant antigens such as the rK39 for serology is an additional diagnostic tool. The rK39 is a kinesin-like protein derived from $L$. infantum (chagasi) which contains a 39 amino acid repeat conserved in $L$. infantum and the closely related $L$. donovani [27]. Serology testing to rK39 is available in qualitative dipsticks and also in quantitative ELISA assays. In this study, sera from $L$. infantum- and L. tropica-infected dogs was positive with the rK39 dipstick, but not sera from L. majorinfected dogs. Positive response to the rK39 dipstick has also been reported from an Iranian young dog with mucocutaneous lesions due to L. tropica [19] suggesting that this test might be useful for the detection of canine $L$. tropica infection. The fact that sera from dogs with $L$. major infection did not react with the rK39 dipstick is interesting but needs further testing with more dogs because although one dog responded to all the crude antigen ELISAs of the three Leishmania spp. but not to rK39, the other dog was not reactive with these three antigens. The absence of a detectable serologic response to $L$. major in one of the dogs can be explained by the fact that this species causes apparently cutaneousrestricted infection in humans and possibly also in dogs, and does not elicit a detectable serologic response in some infected humans [28]. 
The dog with L. major infection (case no. 1) in this study responded well to allopurinol treatment at $10 \mathrm{mg} /$ $\mathrm{kg}$ twice daily, and its skin lesions improved and almost disappeared within 7 weeks of treatment. This successful response to allopurinol treatment is similar to that described for a previous canine L. major case treated with allopurinol and described by us [7]. The response of canine L. tropica to the same treatment regimen was also successful in a previously described case [6] and unfortunately the dog in the current study (case no. 2) was not followed up due to loss of contact with its owners. It should be noted that dogs with L. major and L. tropica infections in the comparative serology study were also treated with allopurinol and this may have affected their serological responses in samples taken after the beginning of treatment by decreasing the amount of antibodies; however, it can be presumed that this would affect the response to different Leishmania spp. antigens similarly.

Although current understanding of the clinical and diagnostic findings and treatment of canine L. major and L. tropica infection is limited by the small number of dogs diagnosed and treated for these infections, this study together with previous reports add to the characterization of disease associated with these two dermatropic species in dogs and to knowledge about its management.

\section{Conclusions}

This study further elucidates the clinical and parasitological findings in canine L. major and L. tropica infections. It suggests that ELISA serology with whole promastigote antigen is not distinctive between $L$. infantum, L. major and L. tropica canine infections and in some cases clinical $L$. major infections may not be seropositive, and it indicates that PCR with DNA sequencing from the affected tissues or blood should be used in dogs to discriminate between infections with these three Old World Leishmania spp.

\section{Additional file}

Additional file 1: Table S1. Optical density readings (OD) of sera from dogs naturally infected with Leishmania infantum, L. major or L. tropica tested by ELISA for reactivity with crude promastigote antigen of these three species. (DOCX $17 \mathrm{~kb}$ )

\section{Abbreviations}

CBC: Complete blood count; ELISA: Enzyme-linked immunosorbent assay; HRM: High resolutions melt; HUSVM: Hebrew University School of Veterinary Medicine; ITS: Internal transcribed spacer; OD: Optical density; PCR: Polymerase chain reaction

\section{Acknowledgments}

The authors thank Drs. Inbar Sever and Shalom Haimowitz and the medical staff of the HUSVM veterinary teaching hospital who treated the dogs reported here. We thank Prof. Charles Jaffe and Dr. Abed Nasereddin for providing some of the Leishmania culture strains. Publication of this paper has been sponsored by Bayer Animal Health in the framework of the $12^{\text {th }}$ CVBD World Forum Symposium.

\section{Funding}

This study was funded by the author's own resources. No funding body had influence on the design of the study, collection, analysis and interpretation of the data.

\section{Availability of data and materials}

All data generated or analysed during this study are included in this published article. Representative sequences were submitted to the GenBank database under accession numbers KY524299 and KY524300.

\section{Authors' contributions}

GB collected the data, sampled the dogs and wrote the manuscript; YNB and MG performed serology; YNB performed PCR and DNA sequencing and analyzed the data; DYL isolated L. tropica and grew the parasite in culture. All authors read and approved the final manuscript.

\section{Competing interests}

The authors declare that they have no competing interests.

\section{Ethics approval}

This study was carried out in accordance with the Hebrew University ethic regulations for experimentation in animals. The study involved exclusive use of samples taken as a part of the animal's diagnostic procedure by attending veterinarians.

Received: 13 February 2017 Accepted: 20 February 2017

Published online: 13 March 2017

\section{References}

1. Postigo JA. Leishmaniasis in the world health organization eastern Mediterranean region. Int J Antimicrob Agents. 2010;36 Suppl 1:S62-5.

2. Ntais P, Sifaki-Pistola D, Christodoulou V, Messaritakis I, Pratlong F, Poupalos G, et al. Leishmaniases in Greece. Am J Trop Med Hyg. 2013;89:906-15.

3. Jaffe $\mathrm{CL}$, Baneth $\mathrm{G}$, Abdeen ZA, Schlein Y, Warburg A. Leishmaniasis in Israe and the Palestinian authority. Trends Parasitol. 2004;20:328-32.

4. Gandacu D, Glazer Y, Anis E, Karakis I, Warshavsky B, Slater P, et al. Resurgence of cutaneous leishmaniasis in Israel, 2001-2012. Emerg Infect Dis. 2014; 20:1605-11.

5. Bamorovat M, Sharifi I, Dabiri S, Mohammadi MA, Fasihi Harandi M, Mohebali $\mathrm{M}$, et al. Leishmania tropica in stray dogs in southeast Iran. Iran J Public Health. 2015:44:1359-66.

6. Baneth G, Zivotofsky D, Nachum-Biala Y, Yasur-Landau D, Botero AM. Mucocutaneous Leishmania tropica infection in a dog from a human cutaneous leishmaniasis focus. Parasit Vectors. 2014;7:118.

7. Baneth G, Nachum-Biala Y, Shabat Simon M, Brenner O, Gaier S, Rojas A, et al. Leishmania major infection in a dog with cutaneous manifestations. Parasit Vectors. 2016:9:246.

8. Nicolas L, Milon G, Prina E. Rapid differentiation of Old world Leishmania species by LightCycler polymerase chain reaction and melting curve analysis. J Microbiol Methods. 2002;51:295-9.

9. Talmi-Frank D, Nasereddin A, Schnur LF, Schönian G, Töz SO, Jaffe CL, et al. Detection and identification of old world Leishmania by high resolution melt analysis. PLoS Negl Trop Dis. 2010;4:e581.

10. Yasur-Landau D, Jaffe CL, David L, Baneth G. Allopurinol resistance in Leishmania infantum from dogs with disease relapse. PLoS Negl Trop Dis. 2016:10:e0004341.

11. Jaffe $C L$, Keren E, Nahary O, Rachamim N, Schnur L. Canine visceral leishmaniaisis at Wadi Hamam, in Israel. Trans R Soc Trop Med Hyg. 1988:82:853-9.

12. Tabar MD, Altet L, Francino O, Sánchez A, Ferrer L, Roura X. Vector-borne infections in cats: molecular study in Barcelona area (Spain). Vet Parasitol. 2008;151:332-6

13. Peleg O, Baneth G, Eyal O, Inbar J, Harrus S. Multiplex real-time qPCR for the detection of Ehrlichia canis and Babesia canis vogeli. Vet Parasitol. 2010;173: 292-9.

14. Peters W, Elbihari S, Liu C, Le Blanca SM, Evans DA, Killick-Kendrick R, et al. Leishmania infecting man and wild animals in Saudi Arabia. 1. General survey. Trans R Soc Trop Med Hyg. 1985:79:831-9.

15. Elbihari S, Cheema AH, El-Hassan AM. Leishmania infecting man and wild animals in Saudi Arabia. 4. Canine cutaneous leishmaniasis in the eastern province. Trans R Soc Trop Med Hyg. 1987;81:925-7. 
16. Morsy TA, Schnur LF, Feinsod FM, Salem AM, Wahba MM, el Said SM. Natural infections of Leishmania major in domestic dogs from Alexandria, Egypt. Am J Trop Med Hyg. 1987;37:49-52.

17. Guessous-Idrissi N, Berrag B, Riyad M, Sahibi H, Bichichi M, Rhalem A. Leishmania tropica: etiologic agent of a case of canine visceral leishmaniasis in northern Morocco. Am J Trop Med Hyg. 1997;57:172-3.

18. Lemrani M, Nejjar R, Pratlong F. A new Leishmania tropica zymodeme causative agent of canine visceral leishmaniasis in northern Morocco. Ann Trop Med Parasitol. 2002;96:637-8.

19. Mohebali M, Malmasi A, Hajjaran H, Jamshidi S, Akhoundi B, Rezaei M, et al. Disseminated leishmaniasis caused by Leishmania tropica in a puppy from Karaj, Central Iran. Iran J Parasitol. 2011;6:69-73.

20. Dereure J, Rioux JA, Gallego M, Perières J, Pratlong F, Mahjour J, et al. Leishmania tropica in Morocco: infection in dogs. Trans R Soc Trop Med Hyg. 1991:85:595.

21. Sacks DL, Kenney RT, Kreutzer RD, Jaffe CL, Gupta AK, Sharma MC, et al. Indian kala-azar caused by Leishmania tropica. Lancet. 1995;345:959-61.

22. Krayter L, Bumb RA, Azmi K, Wuttke J, Malik MD, Schnur LF, et al. Multilocus microsatellite typing reveals a genetic relationship but, also, genetic differences between Indian strains of Leishmania tropica causing cutaneous leishmaniasis and those causing visceral leishmaniasis. Parasit Vectors. 2014;7:123.

23. Solano-Gallego L, Miró G, Koutinas A, Cardoso L, Pennisi MG, Ferrer L, Bourdeau $P$, et al. LeishVet guidelines for the practical management of canine leishmaniosis. Parasit Vectors. 2011;4:86.

24. Barbosa-De-Deus R, Dos Mares-Guia ML, Nunes AZ, Costa KM, Junqueira RG, Mayrink W, et al. Leishmania major-like antigen for specific and sensitive serodiagnosis of human and canine visceral leishmaniasis. Clin Diagn Lab Immunol. 2002;9:1361-6.

25. Ribeiro FC, Schubach Ade O, Mouta-Confort E, Pacheco TM, Madeira Mde F, Abboud LC, et al. Use of ELISA employing homologous and heterologous antigens for the detection of $\lg G$ and subclasses (lgG1 and $\lg G 2$ ) in the diagnosis of canine visceral leishmaniasis. Rev Inst Med Trop Sao Paulo. 2011;53:283-9.

26. Vale AM, Fujiwara RT, da Silva Neto AF, Miret JA, Alvarez DC, da Silva JC, et al. Identification of highly specific and cross-reactive antigens of Leishmania species by antibodies from Leishmania (Leishmania) chagasi naturally infected dogs. Zoonoses Public Health. 2009:56:41-8.

27. Maia Z, Lírio M, Mistro S, Mendes CM, Mehta SR, Badaro R. Comparative study of rK39 Leishmania antigen for serodiagnosis of visceral leishmaniasis: systematic review with meta-analysis. PLoS Negl Trop Dis. 2012;6:e1484.

28. Silvestre R, Santarém N, Teixeira L, Cunha J, Schallig H, Cordeiro-da-Silva A. Evaluation of Leishmania species reactivity in human serologic diagnosis of leishmaniasis. Am J Trop Med Hyg. 2009;81:202-8.

\section{Submit your next manuscript to BioMed Central and we will help you at every step:}

- We accept pre-submission inquiries

- Our selector tool helps you to find the most relevant journal

- We provide round the clock customer support

- Convenient online submission

- Thorough peer review

- Inclusion in PubMed and all major indexing services

- Maximum visibility for your research

Submit your manuscript at www.biomedcentral.com/submit

) Biomed Central 